\title{
ECG Source Location Clustering Based on Position Vectors and Forward Transfer Matrices
}

\author{
Takano N, H-G Puurtinen, M Rautiainen, \\ J Hyttinen, J Malmivuo \\ Ragnar Granit Institute, Tampere University of Technology, \\ Tampere, Finland
}

\begin{abstract}
Heart model segmentation methods concerning ECG source-to-measurement forward transfer modeling are discussed. The k-means clustering technique was adopted to classify all discrete points forming a heart model with respect to their position vectors or source-to-measurement transfer matrices. The clusters were formed in heart models of end-systolic and end-diastolic cardiac phases. The minimum number of clusters for different lead systems, cardiac phases and in volume conductor models determined for least square error approximation of nonclustered (original) transfer model are tabulated. These numbers suggested that the number current dipole sources could be reduced to less than $10 \%$ of that of the source locations. Some of the heart models segmented by the resulting clusters are presented at the end of this article.
\end{abstract}

\section{Introduction}

Since sensitivities of ECG leads are dependent on ECG source locations, ECG source-to-measurement transfer matrices in forward transfer models also vary by the source locations. In order to analyze such sensitivity distributions and/or to reduce the number of sources in forward transfer model, the present study applies the $k$-means clustering technique to the transfer matrices or position vectors of the source locations, and approximates the transfer matrices in each cluster by one matrix. There are already definitions of segmentations of the left ventricle [1], but they are based on anatomical features for myocardial infarction studies, not specially for forward problem of ECG. The approach introduced here is mathematical one, and can be applied to the entire heart.

\section{Theory and method}

\subsection{Source-to-measurement transfer models}

In ECG source-to-measurement forward transfer models, the measurements are computed by liner transformation of ECG sources. Each solution can be given as a vector comprising ECG voltages in an ECG lead system of multiple leads. Current dipole sources are considered in the present study.

\subsubsection{Non-clustered model}

In a non-clustered model, the sources at locations in a heart model are transformed into measurements:

$$
\mathbf{v}=\sum_{l=1}^{L} \mathbf{C}_{l} \mathbf{u}_{l},
$$

$\mathbf{v}$ : forward solution in non-clustered model;

$l:$ index of source location;

$L:$ the number of source locations;

$\mathbf{C}_{l}$ : source-to-measurement transfer matrix at location $l$;

$\mathbf{u}$ : current dipole source vector.

\subsubsection{Clustered model}

In a clustered model, ECG source locations are classified into subsets, and sources at locations in each subset can be summed up before the transformations:

$$
\hat{\mathbf{v}}=\sum_{k=1}^{K} \hat{\mathbf{C}}_{k} \sum_{l \in \mathcal{K}_{k}} \mathbf{u}_{l},
$$

$\hat{\mathbf{v}}$ : forward solution in clustered model using $\hat{\mathbf{C}}_{k=1: K}$;

$k$ : index of mutually associated subset and cluster;

$K$ : the number of subsets or of clusters;

$\hat{\mathbf{C}}_{k}$ : transfer matrix optimized for cluster $k$;

$\mathcal{K}$ : subset of source locations.

The sources to be considered in a clustered model is reduced in such a sense that $K<L$. 


\subsection{LS approximation of transfer matrices}

$\hat{\mathbf{C}}_{k=1: K}$ of clusters are optimized for the least squared (LS) error approximations of $\mathbf{C}_{l \in \mathcal{K}_{k}}$ for each $k$. They are obtained by numerical averaging of $\mathbf{C}_{l}$ in each $\mathcal{K}_{k}$ :

$$
\hat{\mathbf{C}}_{k}=\frac{1}{L_{k}} \sum_{l \in \mathcal{K}_{k}} \mathbf{C}_{l}, k=1: K,
$$

where $L_{k}$ is the number of location indices in $\mathcal{K}_{k}$.

As an evaluation of the approximations, approximation error ratio, $\xi_{K}$, is defined here. It is essentially the summation of squared Euclidean norms of the approximation errors, $\mathbf{C}_{l}-\hat{\mathbf{C}}_{k}$ :

$$
\xi_{K}=\frac{\sum_{k=1}^{K} \sum_{l \in \mathcal{K}_{k}}\left\|\mathbf{C}_{l}-\hat{\mathbf{C}}_{k}\right\|_{E}^{2}}{\sum_{l=1}^{L}\left\|\mathbf{C}_{l}\right\|_{E}^{2}}
$$

The denominator is for such a normalization that $0 \leq$ $\xi_{K} \leq 1$. The suffix of $\xi_{K}$ indicates the number of clusters used for the approximations.

\section{3. $\quad k$-means clustering}

In order to form subsets $\mathcal{K}_{k=1: K}$, the $k$-means clustering technique [2] was employed. It classifies indices $l=$ $1: L$ of data samples, denoted here by $\mathbf{x}_{l}$, of interest to form clusters. Successfully formed clusters exhibit high homogeneity of $\mathbf{x}_{l}$ within cluster and high heterogeneity between clusters. This method iterates the steps listed here: 1. Centroid, $\dot{\mathbf{x}}_{k}$, is calculated in each cluster:

$$
\dot{\mathbf{x}}_{k}=\frac{1}{L_{k}} \sum_{l \in \mathcal{K}_{k}} \mathbf{x}_{l}, k=1: K,
$$

2. $l=1: L$ are classified by "closeness" to the centroids:

$$
\mathcal{K}_{k}=\left\{l \mid k=\arg \min _{k^{\prime}}\left\|\mathbf{x}_{l}-\dot{\mathbf{x}}_{k^{\prime}}\right\|_{E}\right\}, k=1: K,
$$

3. If $\dot{\mathbf{x}}_{k=1: K}$ have converged, quit, otherwise return to 1 .

Two types of clusters were formed by characteristics of source locations as listed below.

Transfer matrix-based clustering : $\mathbf{C}_{l=1: L}$ in non-clustered model were used as the characteristic $\mathrm{x}$ seen in (5) and (6); in this clustering (5) is identical to (3);

Position vector-based clustering : Position vectors in a Cartesian coordinate system of locations, $l=1: L$, were clustered, $\hat{\mathbf{C}}_{k=1: K}$ of these clusters being determined and evaluated by (3) and (4).

\subsection{Experiment procedure}

Each clustering process commenced with $K=1$. After the clusters converged, $K$ was incremented by 1 and the clustering was executed again. Transfer matrix-based clustering process stopped when $\xi_{K} \leq 0.01$ was obtained, and position vector-based stopped when $\xi_{K} \leq 0.1$.

\section{Study material}

\subsection{Thorax-heart models}

The transfer matrices were computed in the volume conductor thorax models listed here:

$i$-sys : inhomogeneous, end-systolic;

i-dia : inhomogeneous, end-diastolic;

h-sys : homogeneous, end-systolic;

h-dia : homogeneous, end-diastolic.

Two of them are inhomogeneous conductor models constructed from magnetic resonance images for the finite difference method (FDM) computing, and the other assumed an infinite extent of homogeneous conductivity. The origin of the four is i-sys model, which contains a heart model of end-systolic cardiac phase. Except for the heart model of end-diastolic phase, i-dia model is identical with i-sys. All FDM grid nodes forming a heart model were used as source locations. The end-systolic heart model was formed by 12032 location points and the end-diastolic heart model by 13120 . In the homogeneous models electrodes and source locations were set the same as the inhomogeneous models.

\subsection{Lead systems}

Since the transfer matrix is formed for a specific lead system, clustering analysis was performed for several lead systems, including these in the list:

bsm120 : A body surface mapping (BSM) lead system [3] comprising 120 unipolar leads;

$k r n 5$ : 5 leads selected by Kornreich's group for multiple diagnosis [4], a subset of bsm120;

limb3 : 3 unipolar limb leads;

prec6 : 6 precordial leads in the standard leads;

limb3 + prec6 : combination of limb3 and prec6;

std12 : standard 12 ECG leads, including prec6, and augmented limb and bipolar limb leads;

frank7 : unipolar leads at 7 electrode sites defined for Frank vector leads, using the Wilson central terminal;

frank : Frank vector leads.

\section{Results}

As $K$ was incremented, $\xi_{K}$ decreased monotonously. The minimum $K$ to obtain certain values of $\xi_{K}$ are summarized in Table 1, which may suggest the number of clusters (or sources) necessary for constructing forward transfer models as reduced versions of the original (nonclustered) model. Position vector-based clusters for $\xi_{K} \leq$ 0.1 were so many as the transfer matrix-based clusters for far lower $\xi$. $\min K$ did not always reflect the number of leads or electrode sites in the lead systems. 
Cluster partitions in a heart model differed by clustering methods and conductivity models even if $\xi_{K}$ or $K$ are the same, for instance $\xi_{K} \leq 0.1$ and $K \approx 750$ seen in Figure 1. The position vector-based clusters were almost equally sized in each clustered model while the transfer matrixbased clusters were smaller in regions closer to electrodes. In the inhomogeneous models with transfer matrix-based clustering, locations near to and far from a boundary of the heart were often classified into different clusters; such partitions were not seen in the homogeneous models. Bear in mind, identical sources at different locations in each cluster produce approximately the same measurements.

\section{Conclusion}

Although the position vector-based clustering, as it is defined, made spatially clear partitions in the heart models, it was not so efficient for ECG source reduction as the transfer matrix-based clustering. The transfer matrixbased clusters had their sizes varied by regions in the heart and formed intricate spatial partitions, which were also affected by inhomogeneous conductivities in the thoraxheart model. It was also observed that increase or decease of the number of clusters sufficient for forward transfer model could not be expected directly from change in the number of leads or electrodes in the lead system.

\section{References}

[1] Startt/Selvester RH, Wagner GS, Ideker RE. Myocardial infarction. In Macfarlane PW, Lawrie TDV (eds.), Comprehensive Electrocardiography: Theory and Practice in Health and Disease, volume 1, first edition. Elmsford, New York, U.S.A.: Pergamon Press, 1989; 565-629.

[2] Rabiner LR, Juang BH. Fundamentals of Speech Recognition. Englewood Cliffs, New Jersey, U.S.A.: Prentice-Hall, 1993; 122-132.

[3] Montague TJ, Smith ER, Cameron DA, Rautaharju PM, Klassen GA, Felmington CS, Horaceck BM. Isointegral analysis of body surface maps: Surface distribution and temporal variability in normal subjects. Circulation May 1981;63(5):1166-1172.

[4] Korneich F, Montague TJ, Pentti Rautaharju MK, Horaceck MB, Taccardi B. Multigroup diagnosis of body surface potential maps. Journal of Electrocardiology 1989;22 Supplement:169-178.

Address for correspondence:

TAKANO Noriyuki

Ragnar Granit Institute, Tampere University of Technology

P.O. Box 692 FIN-33101 Tampere Finland

tel: $+358-3-31152524$

fax: $+358-3-31152162$

noriyuki.takano@tut.fi

\begin{tabular}{|c|c|c|c|c|c|c|}
\hline \multicolumn{7}{|c|}{ i-sys model, $L=12032$ source locations } \\
\hline \multirow[b]{2}{*}{ Lead Config } & \multirow[b]{2}{*}{$N_{L} / N_{E}$} & \multirow{2}{*}{ PVB } & \multicolumn{4}{|c|}{ TMB } \\
\hline & & & 0.1 & 0.05 & 0.03 & 0.01 \\
\hline bsm120 & $120 / 120$ & 718 & 77 & 291 & 781 & 1899 \\
\hline krn5 & $5 / 8$ & 846 & 24 & 94 & 222 & 997 \\
\hline limb3 & $3 / 3$ & 41 & 4 & 19 & 64 & 572 \\
\hline prec6 & $6 / 9$ & 1008 & 32 & 109 & 260 & 990 \\
\hline limb3 + prec6 & 9/9 & 958 & 33 & 128 & 274 & 1077 \\
\hline $\operatorname{std} 12$ & $12 / 9$ & 884 & 33 & 138 & 312 & 1268 \\
\hline frank7 & $7 / 9$ & 1054 & 25 & 94 & 237 & 1055 \\
\hline frank & $3 / 7$ & 498 & 15 & 65 & 195 & 1192 \\
\hline \multicolumn{7}{|c|}{ i-dia model, $L=13120$ source locations } \\
\hline Lead Config & $N_{L} / N_{E}$ & 0.1 & 0.1 & 0.05 & 0.03 & 0.01 \\
\hline bsm120 & $120 / 120$ & 1318 & 100 & 349 & 700 & 2033 \\
\hline krn5 & $5 / 8$ & 2365 & 27 & 94 & 225 & 959 \\
\hline limb3 & $3 / 3$ & 113 & 7 & 23 & 70 & 666 \\
\hline prec6 & $6 / 9$ & 1877 & 30 & 127 & 286 & 965 \\
\hline limb3 + prec6 & 9/9 & 1730 & 33 & 120 & 297 & 1019 \\
\hline $\operatorname{std12}$ & $12 / 9$ & 1551 & 35 & 154 & 327 & 1196 \\
\hline frank7 & $7 / 9$ & 1743 & 26 & 91 & 202 & 933 \\
\hline frank & $3 / 7$ & 994 & 16 & 63 & 194 & 1229 \\
\hline \multicolumn{7}{|c|}{$\overline{\text { h-sys model, } L=12032 \text { source locations }}$} \\
\hline Lead Config & $N_{L} / N_{E}$ & 0.1 & 0.1 & 0.05 & 0.03 & 0.01 \\
\hline bsm120 & $120 / 120$ & 37 & 24 & 66 & 147 & 764 \\
\hline krn5 & $5 / 8$ & 39 & 19 & 53 & 115 & 601 \\
\hline limb3 & $3 / 3$ & 1 & & 2 & 4 & 15 \\
\hline prec6 & $6 / 9$ & 55 & 20 & 58 & 133 & 674 \\
\hline limb3 + prec6 & $9 / 9$ & 54 & 20 & 57 & 136 & 669 \\
\hline $\begin{array}{l}\text { std12 } \\
\text { sta }\end{array}$ & $12 / 9$ & 48 & 19 & 55 & 127 & 662 \\
\hline frank7 & $7 / 9$ & 54 & 21 & 58 & 128 & 669 \\
\hline frank & $3 / 7$ & 11 & 4 & 14 & 32 & 198 \\
\hline \multicolumn{7}{|c|}{ h-dia model, $L=13120$ source locations } \\
\hline Lead Config & $N_{L} / N_{E}$ & 0.1 & 0.1 & 0.05 & 0.03 & 0.01 \\
\hline bsm120 & $120 / 120$ & 53 & 29 & 82 & 187 & 948 \\
\hline krn5 & $5 / 8$ & 52 & 21 & 56 & 128 & 627 \\
\hline limb3 & $3 / 3$ & 1 & & & 4 & 17 \\
\hline prec6 & $6 / 9$ & 72 & 26 & 70 & 164 & 782 \\
\hline limb3 + prec6 & 9/9 & 69 & 26 & 69 & 169 & 767 \\
\hline std12 & $12 / 9$ & 68 & 25 & 69 & 156 & 742 \\
\hline frank7 & $7 / 9$ & 86 & 27 & 75 & 168 & 683 \\
\hline frank & $3 / 7$ & 31 & 8 & 23 & 60 & 280 \\
\hline
\end{tabular}

Table 1. The minimum number of clusters, $K$, to obtain approximation error ratio $\xi_{K}$ of transfer matrices $\leq 0.1$ by the position vector-based (PVB) clustering and 0.1 to 0.01 by the transfer matrix-based (TMB) clustering in isys, i-dia, h-sys, h-dia thorax-heart models each containing a heart model formed by $L$ source locations. These minimum numbers were evaluated for the lead systems whose number of leads and electrode sites are indicated by $N_{L}$ and $N_{E}$, respectively, in the second column. 
\begin{tabular}{l|l} 
Inhomogeneous thorax model with end-systolic heart model & Homogeneous thorax model with end-systolic heart model
\end{tabular}

position vector-based clusters for $\xi_{K} \leq 0.1, K=718$

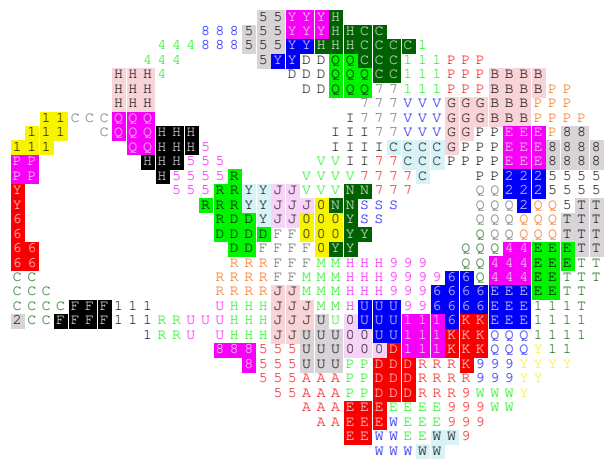

transfer matrix-based clusters for $\xi_{K} \leq 0.1, K=77$

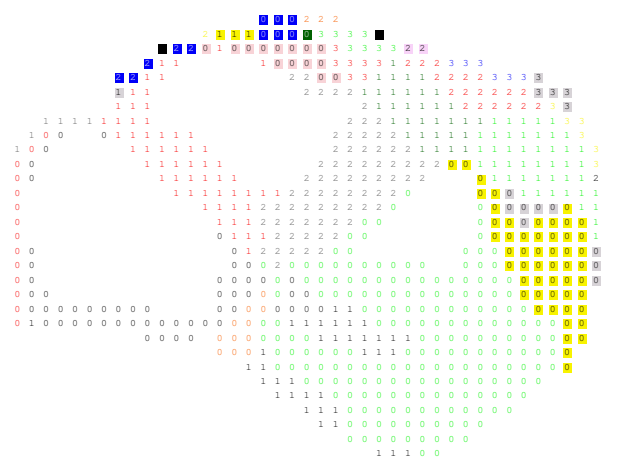

transfer matrix-based clusters for $\xi_{K} \leq 0.03, K=781$

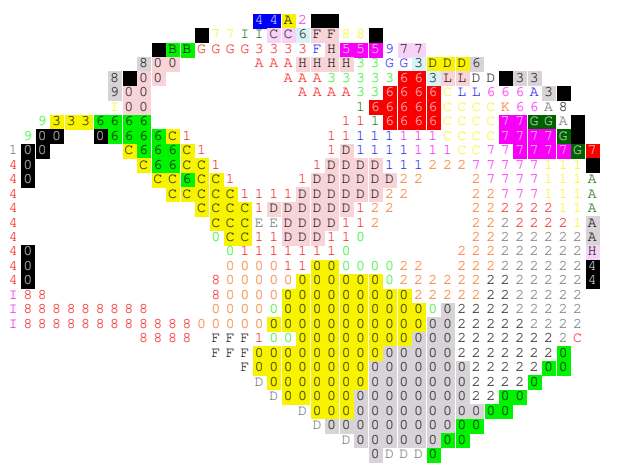

\section{position vector-based clusters for $\xi_{K} \leq 0.1, K=37$}

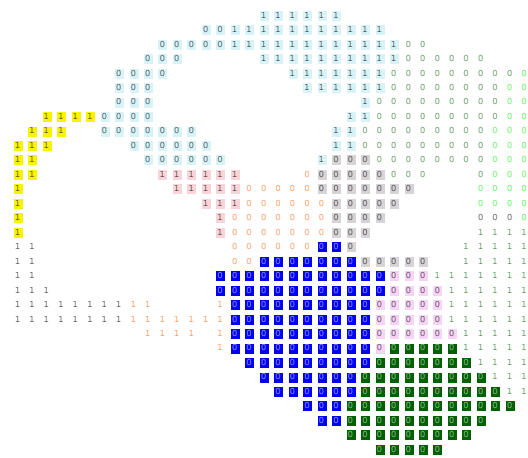

transfer matrix-based clusters for $\xi_{K} \leq 0.1, K=24$

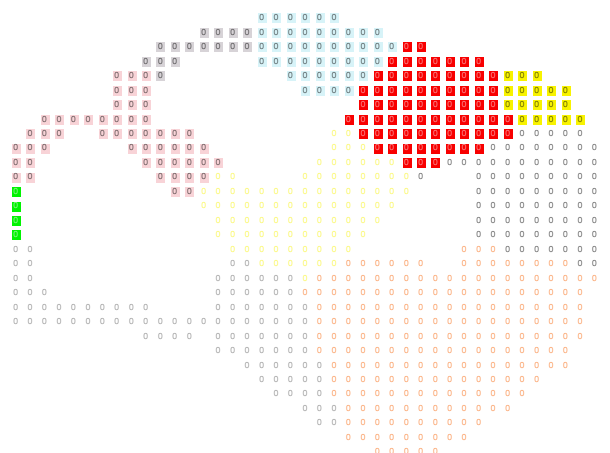

transfer matrix-based clusters for $\xi_{K} \leq 0.01, K=764$

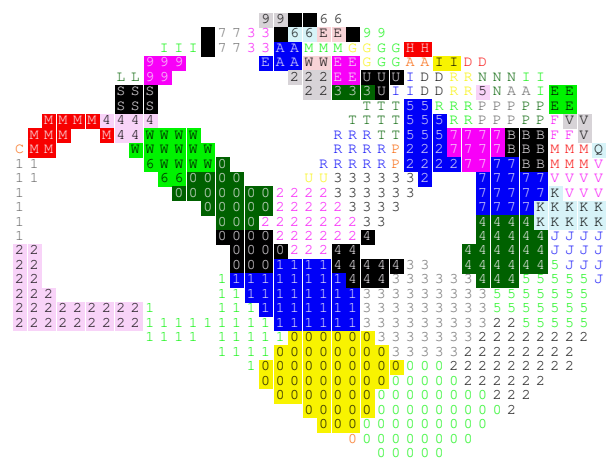

Figure 1. Transverse views of clustered heart models (anterior side is up, and left side of the body is right-hand side of the slice). On the left-hand side are results in i-sys model, on the right-hand side are those in h-sys. These indicate classifications of locations according to the minimum $K$ of clusters to obtain $\xi_{K}$ less than or equal to a given value written on each slice picture regarding approximation of $\mathbf{C}$ in non-clustered model for bsm120 lead system (see also Table 1). Combinations of character, fore- and background colors indicate clusters into which the locations are classified. These combinations are consistent in each clustering result only. They do not indicate any characteristic of the clusters, except for that the squares filled in black in the anterior regions indicate clusters each consisting of only one location. 